\title{
Splash Jet Generated by Collision of Two Liquid Wedges
}

\author{
By Y. A. SEMENOV ${ }^{1}$ ， G. X. WU ${ }^{1} \dagger$ AND J.M. OLIVER ${ }^{2}$ \\ ${ }^{1}$ Department of Mechanical Engineering, University College London, London WC1E 6BT, UK \\ ${ }^{2}$ Mathematical Institute, University of Oxford, 24-29 St Giles, Oxford OX1 3LB, UK
}

(Received ?? and in revised form ??)

A complete nonlinear self-similar solution that characterizes the impact of two liquid wedges symmetric about the velocity direction is obtained assuming the liquid to be ideal and incompressible, with negligible surface tension and gravity effects. Employing the integral hodograph method, analytical expressions for the complex potential and for its derivatives are derived. The boundary-value problem is reduced to two integrodifferential equations in terms of the velocity modulus and angle to the free surface. Numerical results are presented in a wide range of wedge angles for the free surface shapes, streamline patterns, and pressure distributions. It is found that the splash jet may cause secondary impacts. The regions with and without secondary impacts in the plane of the wedges angles are determined.

KEY WORDS: impact of liquids; splash jet; complex velocity potential; free-surface flow.

\section{Introduction}

The formation of a splash following the collisions of liquids and granular materials is a widely observed phenomenon in nature and engineering. Examples include plunging breaking water waves, drops impacting a free surface or a thin film of the same liquid, etc. Splashing may lead to fluid fragmentation and generation of droplets and sprays. These phenomena have common features of liquid defragmentation near the free surface, liquid evaporation and air entrainment, generation of cavitation nuclei, bubbles, secondary drops and sprays. The review of these phenomena over a range of diversified problems was presented by Yarin (2006) who focused on liquid drop impacts on thin liquid layers and dry surfaces. Kiger and Duncan (2012) described the process of air-entrainment, and Thoroddsen et al. (2008) centred the discussions on the initial stage of drop impact when liquid masses came into contact and merge.

The investigation of liquid splashing during the last century was mostly based on experimental observation. Over the last decade new experimental tools such as high-speed video camera technologies Thoroddsen (2002) and the X-ray technique Zhang et al. (2012) were applied to fluid impacts and revealed complex structure of the flow at the initial stage accompanied by fluid fragmentation and the formation of a spray cloud. However, it is still far from giving a thorough insight into this phenomenon, especially at the initial stage. Direct numerical simulations of splashing for drops impacting on a liquid layer were performed by Weiss and Yarin (1999) and Davidson (2002) based on the boundary integral method with the inclusion of surface tension forces. Incompressible Navier-Stokes

$\dagger$ Email address for correspondence: g.wu@ucl.ac.uk 
equations with surface tension were solved numerically by Josserand \& Zaleski (2003). The initial stage of high-velocity droplet impact on a shallow water layer was investigated by Howison et al. (2005) and Oliver (2007) using the method of matched asymptotic expansions. A numerical investigation of splashing and wave breaking processes using the SPH method was performed by Landrini et al. (2007).

A related free boundary problem is that investigated by Keller \& Miksis (1983) and Keller et al. (2002). They considered two wedges of liquid initially at rest and touching at their vertices. For later times the free surface flow is driven by the surface tension only. They introduced self-similar variables

$$
x=X\left(\frac{\rho}{\sigma t^{2}}\right) \quad \text { and } \quad y=Y\left(\frac{\rho}{\sigma t^{2}}\right),
$$

where $\rho$ is the density and $\sigma$ is the surface tension coefficient. A system of integrodifferential equations obtained includes two equations with respect to the free surface shape and the velocity potential, which were solved numerically.

In this study, we investigate splash jet formation at the initial stage following an impact between two liquids of the same density using velocity potential theory with fully nonlinear boundary conditions, based on the assumption that the liquid is inviscid and incompressible. When the gravity and surface tension effects are ignored, the flow becomes self-similar. Such a formulation makes it possible to determine the flow topology at the initial stage of the impact when two liquid masses collide and form a splash jet. The integral hodograph method (Semenov \& Cummings (2006)) is employed to derive analytical expressions for the complex-velocity potential, the complex-conjugate velocity, and the mapping function. They are all defined in the first quadrant of a parameter plane, in which the original boundary value problem is reduced to two integro-differential equations in terms of the velocity magnitude and the velocity angle to the liquid boundary. They are solved numerically using the method of successive approximations. The results are presented through streamlines and the pressure distributions along the line of symmetry and near the root of the splash jet. The obtained results have given some insights into the extremely complex flow structure observed in experiments.

\section{Formulation and analysis}

We consider the problem in which two symmetric liquid wedges of half-angles $\alpha_{+}$and $\alpha_{-}$move in opposite directions parallel to their symmetry lines with velocity $V_{0}$ and $V_{D}$, respectively, and they meet head on at point $A$ at time $t=0$, where the origin of the Cartesian coordinate system of $X-Y$ is chosen. A sketch of the problem and the definitions of the geometric parameters are shown in figure $1 a$. It is assumed that the $Y$-axis along the symmetry line of the liquid wedges. Due to the collision, a splash jet appears with the tip at point $C$ as well as a stagnation point on the $Y$-axis. The velocity at point $P, V_{0}$, is used as a reference velocity and velocity $V_{D}$ is determined from the solution of the problem to satisfy the condition that the location of the stagnation point occurs at point $A$.

For impact of two liquid wedges with constant velocities, the time-dependent problem in the physical plane $Z=X+i Y$ has no explicit length scale. In such a case the shape of the flow pattern is expected to remain unchanged although the size varies with time. As a result, the problem becomes time independent in the self similar plane $z=x+i y$ which are defined as $x=X /\left(V_{0} t\right), y=Y /\left(V_{0} t\right)$. When $V_{0}$ is used as a reference velocity, the velocity magnitude of the upper liquid wedge at infinity, point $P$, in the self-similar plane is unity $\left(v_{0}=1\right)$. The complex velocity potential $W(Z, t)=\Phi(X, Y, t)+i \Psi(X, Y, t)$ 

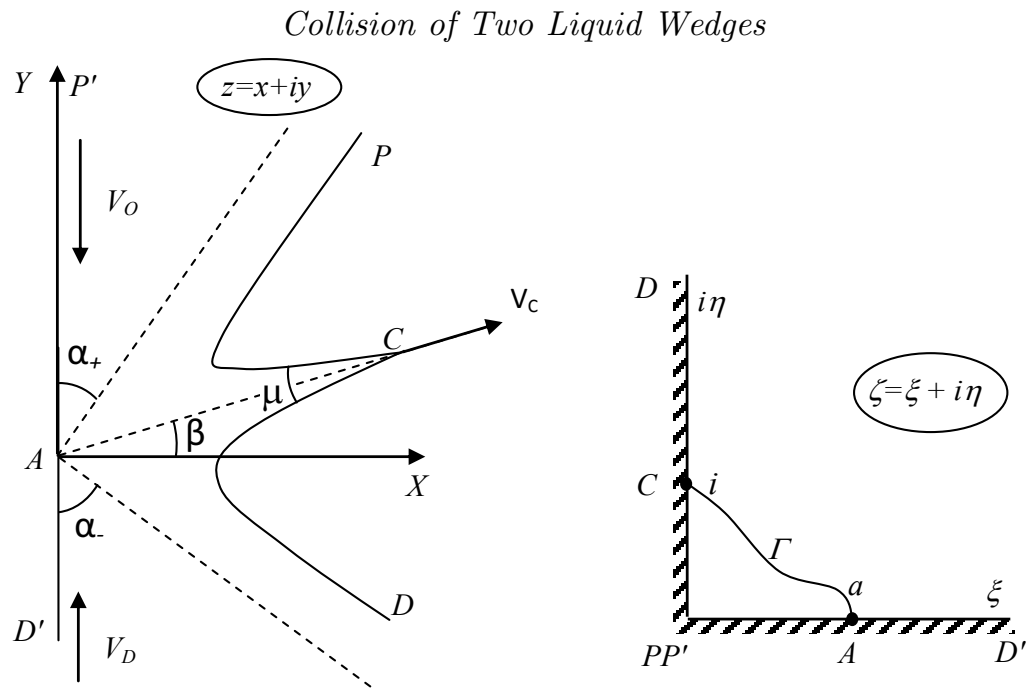

FiguRE 1. (a) sketch of the collision of two liquid wedges; the dotted lines are the initial free surfaces of the wedges at the moment of impact at the origin; the solid lines are the shape of the free surface at a latter time; $C$ is the tip of the splash jet which trajectory is shown by the dash-dotted line; $(b)$ the parameter plane.

for self-similar flows is written in the form

$$
W(Z, t)=\Phi(X, Y, t)+i \Psi(X, Y, t)=V_{0}^{2} t w(z)=V_{0}^{2} t[\phi(x, y)+i \psi(x, y)]
$$

where $\phi$ and $\psi$ are the velocity potential and the stream function in the self-similar plane.

The problem is now to determine the function $w(z)$ which conformally maps the selfsimilar plane $z$ onto the complex-velocity potential region $w$. Following Joukovskii's method, we choose the first quadrant of the $\zeta$-plane as the parameter region to derive expressions for the nondimensional complex velocity, $v_{x}-i v_{y}=d w / d z$, and for the derivative of the complex potential, $d w / d \zeta$, both as functions of the variable $\zeta=\xi+i \eta$. Once these functions are found, the velocity field and the mapping function $z=z(\zeta)$ are determined as follows

$$
v_{x}(\xi, \eta)-i v_{y}(\xi, \eta)=\frac{d w}{d z}(\zeta), \quad z(\zeta)=z_{A}+\int_{a}^{\zeta} \frac{d z}{d \zeta^{\prime}} d \zeta^{\prime}, \quad \frac{d z}{d \zeta}=\frac{d w}{d \zeta} / \frac{d w}{d z},
$$

\subsection{Expressions for the governing functions}

Conformal mapping allows us to fix three arbitrary points in the parameter region, which are $P, C$, and $D$ as shown in figure $1 b$. The image of point $A(\zeta=a)$ should be determined as a part of the solution of the problem. In this plane, the interval of the imaginary axis $(0<\eta<1, \xi=0)$ corresponds to the free surface $C P$, and the interval $(1<\eta<\infty$, $\xi=0)$ corresponds to the free surface $C D$. The positive real axis $(0<\xi<\infty, \eta=0)$ corresponds to the symmetry line $P^{\prime} D^{\prime}$, or the $Y$ axis.

The boundary-value problem for the complex-velocity function can be formulated in the parameter plane. At this stage, we write the velocity modulus along the free surface, that is along the positive part of the imaginary axis of the $\zeta$-plane as $|d w / d z|_{\zeta=i \eta}=$ $v(\eta), \quad 0<\eta<\infty$. This function will be determined below using the dynamic boundary condition.

In the frame of reference fixed at the stagnation point $A$, the velocity component normal to the symmetry line equals zero, while the tangential component is negative along $A P^{\prime}$ and positive along $A D^{\prime}$. This means that the argument of the complex velocity, 


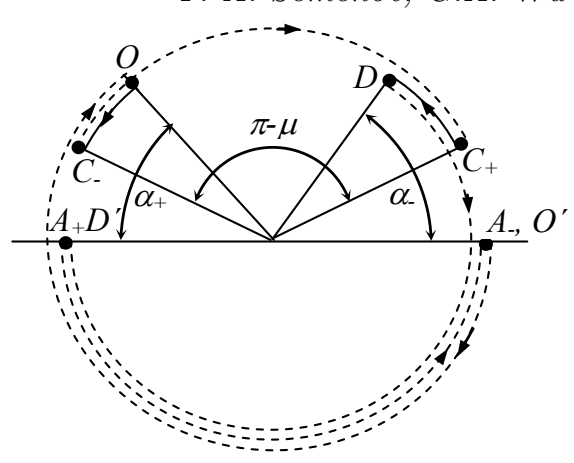

(a)

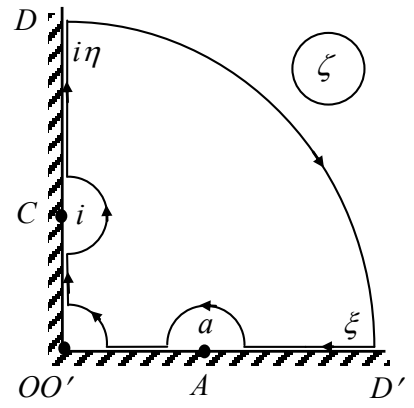

(b)

FIGURE 2. (a) variation of $\theta=\tan ^{-1}\left(v_{n} / v_{\tau}\right)$ along the boundary of the liquid region, (b) the corresponding variation in the parameter plane.

$\chi=\arg (d w / d z)_{\zeta=\xi}$, along the real axis of the parameter region is known as $\chi(\xi)=$ $\pi / 2, \quad 0<\xi<a$, and $\chi(\xi)=-\pi / 2, \quad a<\xi<\infty$.

The problem is then to find the function $(d w / d z)$ in the first quadrant of the parameter plane, which satisfies the given boundary conditions. The integral formula Semenov \& Iafrati (2006) and Semenov \& Cummings (2006)

$$
F(\zeta)=v_{\infty} \exp \left[\frac{1}{\pi} \int_{0}^{\infty} \frac{d \chi}{d \xi} \ln \left(\frac{\zeta+\xi}{\zeta-\xi}\right) d \xi-\frac{i}{\pi} \int_{0}^{\infty} \frac{d \ln v}{d \eta} \ln \left(\frac{\zeta-i \eta}{\zeta+i \eta}\right) d \eta+i \chi\right]
$$

determines the complex function $F(\zeta)$ in the complex plane $\zeta$, which satisfies the given boundary conditions on the real and imaginary axes of the first quadrant: $\chi(\xi)=$ $\arg [F(\zeta)], 0<\xi<\infty, \eta=0$ and $v(\eta)=|F(\zeta)|, 0<\eta<\infty, \xi=0, v_{\infty}=\left.v(\eta)\right|_{\eta=\infty}$, $\chi_{\infty}=\left.\chi(\xi)\right|_{\xi=\infty}$. Applying the above formula for solving the mixed boundary-value problem and evaluating the first integral over the step change of the function $\chi(\xi)$, we finally obtain the expression for the complex velocity in the $\zeta$ - plane as

$$
\frac{d w}{d z}=v_{0}\left(\frac{\zeta-a}{\zeta+a}\right) \exp \left[-\frac{i}{\pi} \int_{0}^{\infty} \frac{d \ln v}{d \eta} \ln \left(\frac{i \eta-\zeta}{i \eta+\zeta}\right) d \eta-i \frac{\pi}{2}\right] .
$$

It can be easily verified that for $\zeta=\xi$ the argument of the RHS of Eq. (2.4) is the function $\chi$, while for $\zeta=i \eta$ the modulus of the RHS of Eq. (2.4) is the function $v(\eta)$, i.e. the above boundary conditions are satisfied.

In order to analyse the behaviour of the velocity potential along the free surface, it is useful to introduce the unit vectors $\vec{n}$ and $\vec{\tau}$ which are normal and tangent to the free surface, respectively. The normal vector is directed from the liquid region outward while along the free surface $s$ increases and the liquid region is on the left (see figure $1 a$ ). With this notation,

$$
d w=\left(v_{s}+i v_{n}\right) d s,
$$

where $v_{s}$ and $v_{n}$ are the tangential and normal velocity components, respectively. Let $\theta$ denote the angle between the velocity vector on the free surface and $\vec{\tau}, \theta=\tan ^{-1}\left(v_{n} / v_{s}\right)$. Eq. (2.5) allows us to determine the argument of the derivative of the complex potential, $\vartheta=\arg (d w / d \zeta)$ 


$$
\begin{aligned}
& \text { Collision of Two Liquid Wedges } \\
& \vartheta(\zeta)=\arg \left(\frac{d w}{d \zeta}\right)=\arg \left(\frac{d w}{d s}\right)+\arg \left(\frac{d s}{d \zeta}\right)=\left\{\begin{array}{l}
\theta, \quad 0<\xi<\infty, \eta=0 \\
\theta+\pi / 2, \quad \xi=0,0<\eta<\infty
\end{array}\right.
\end{aligned}
$$

Now we have to determine the function $\theta(\zeta)$ along the whole fluid boundary, that is, along the real and imaginary axes of the parameter region. The function $\theta$ changes continuously along the parts $P C$ and $C D$ of the free surface, and it changes stepwise upon going around points $P, C, D$ and $A$. Going around the first quadrant of the parameter plane counter clockwise, as shown in figure $2 b$, corresponds to going around the liquid region in the self-similar plane $z$ in the positive direction of the spatial coordinate $s$ (see figure 1a). The corresponding change of the function $\theta$ is shown in figure $2 a$. The continuous change along the free surface is shown by solid lines, while its step changes are shown by dashed lines. The step changes at points $P, C, D$, and $A$, as is seen from figure $2 a$, are $\Delta \theta_{O}=-\left(\pi+\alpha_{+}\right), \Delta \theta_{C}=-\pi+\mu, \Delta \theta_{D}=-\left(\pi+\alpha_{-}\right)$, and $\Delta \theta_{A}=\pi$, respectively.

By introducing the continuous function $\lambda(\varsigma)$ we can write the function $\theta(\varsigma)$ as follows

$$
\theta(\zeta)+\pi= \begin{cases}0, & a<\xi<\infty, \eta=0, \\ \Delta \theta_{A}, & 0<\xi<a, \eta=0, \\ \lambda(\eta)+\Delta \theta_{A}+\Delta \theta_{O}, & \xi=0,0<\eta<1, \\ \lambda(\eta)+\Delta \theta_{A}+\Delta \theta_{O}+\Delta \theta_{C}, & \xi=0,1<\eta<\infty .\end{cases}
$$

where $\lambda(0)=0$.

The problem is then to find the function $d w / d \zeta$ in the first quadrant of the parameter plane which satisfies the boundary condition (2.6). This is a uniform boundary value problem or a problem has the same type of boundary condition. Applying integral formula Semenov \& Iafrati (2006) and Semenov \& Cummings (2006)

$$
\frac{d w}{d \varsigma}=K \exp \left[-\frac{1}{\pi} \int_{0}^{\infty} \frac{d \vartheta}{d \xi} \ln \left(\varsigma^{2}-\xi^{2}\right) d \xi+\frac{1}{\pi} \int_{0}^{\infty} \frac{d \vartheta}{d \eta} \ln \left(\varsigma^{2}+\eta^{2}\right) d \eta+i \vartheta_{\infty}\right]
$$

where $K$ is a real factor, $\vartheta(\zeta)=\arg [d w / d \zeta], 0<\xi<\infty, \eta=0$ and $0<\eta<\infty$, $\xi=0, \vartheta_{\infty}=\vartheta(\zeta)_{\zeta \rightarrow \infty}$, for solving boundary value problem (2.6), (2.7) and evaluating the integrals over each step change of the function $\theta(\zeta)$, we finally obtain the expression for the derivative of the complex potential in the $\zeta$-plane as

$$
\frac{d w}{d \zeta}=K \zeta^{-2 \alpha_{+} / \pi-1} \frac{\left(\zeta^{2}-a^{2}\right)}{\left(\zeta^{2}+1\right)^{1-\mu / \pi}} \exp \left[\frac{1}{\pi} \int_{0}^{\infty} \frac{d \lambda}{d \eta} \ln \left(\zeta^{2}+\eta^{2}\right) d \eta\right] .
$$

where, $K$ is a real factor. Integration of Eq. (2.9) in the parameter region allows us to obtain the function $w=w(\zeta)$ that conformally maps the parameter region onto the corresponding region in the complex potential plane:

$$
w(\zeta)=w_{A}+K \int_{a}^{\zeta} \zeta^{\prime\left(-2 \alpha_{+} / \pi-1\right)} \frac{\left(\zeta^{\prime 2}-a^{2}\right)}{\left(\zeta^{\prime 2}+1\right)^{1-\mu / \pi}} \exp \left[\frac{1}{\pi} \int_{0}^{\infty} \frac{d \lambda}{d \eta} \ln \left(\zeta^{\prime 2}+\eta^{2}\right) d \eta\right] d \zeta^{\prime}
$$

where $w_{A}$ is a constant.

Dividing (2.9) by (2.4), we can obtain derivative of the mapping function $d z / d \zeta$ whose integration (Eq.(2.2)) gives the mapping function $z=z(\zeta)$. Eqs. (2.4) and (2.9) include the parameters $K$ and $a$, and the functions $v(\eta)$ and $\lambda(\eta)$ to be determined from physical considerations, and the dynamic and kinematic boundary conditions. At time $t=0$, the tip of the splash jet, point $C$, and the stagnation point $A$ at the origin coincide. The tip 
of the splash jet moves with the velocity of the liquid at point $C$ having the magnitude $v_{C}=\left.v(\eta)\right|_{\eta=1}$ and the angle $\beta=-\left.\arg (d w / d z)\right|_{\zeta=i}$ with the $x$-axis. Thus we can write the following equation,

$$
z_{C}=\int_{\Gamma} \frac{d z}{d \zeta} d \zeta=v_{C} e^{i \beta}
$$

where $\Gamma$ is an arbitrary contour in the parameter plane connecting points $A$ and $C$ as shown in figure $1 b$. The last equation makes it possible to determine the parameters $K$ and $a$ if the functions $v(\eta)$ and $\lambda(\eta)$ are known.

The originality of the integral hodograph method lies in combination of two methods, namely the boundary integral and hodograph methods. The integral formulae (2.3) and (2.8) give the possibility to find expressions for the complex velocity and for the derivative of the complex potential defined in the parameter plane and extract all the flow singularities in explicit form which are consistent with the hodograph method. These expressions may contain unknown non-singular functions, namely the velocity magnitude and the slope of the solid surface which are determined from derived integral equations which are consistent with the boundary integral method.

\subsection{Dynamic and kinematic boundary conditions}

The dynamic and kinematic boundary conditions for an arbitrary self-similar flow were derived by Semenov \& Iafrati (2006) exploiting the Bernoulli equation and the fact that along the free surface the acceleration of liquid particles is orthogonal to the free boundary. These equations have the form

$$
\begin{gathered}
\frac{d \theta}{d s}=\frac{v+s \cos \theta}{s \sin \theta} \frac{d \ln v}{d s}, \\
\frac{1}{\tan \theta} \frac{d \ln v}{d s}=\frac{d}{d s}\left[\arg \left(\frac{d w}{d z}\right)\right],
\end{gathered}
$$

Multiplying both sides of Eqs. (2.12) and (2.13) by $d s / d \eta=|d z / d \zeta|_{\zeta=i \eta}$ and taking into account that $d \theta / d s=d \lambda / d s$, we obtain the following integro-differential equation for the function $\lambda(\eta)$ :

$$
\frac{d \lambda}{d \eta}=\frac{v+s \cos \theta}{s \sin \theta} \frac{d \ln v}{d \eta}
$$

where $s=s(\eta)$ is obtained by integration of the expression $|d z / d \zeta|_{\zeta=i \eta}$ along the imaginary axis of the parameter plane. Determining the argument of the complex velocity from Eq. (2.4) and substituting the result into Eq. (2.13), the following integral equation for the function $d(\ln v) / d \eta$ is obtained

$$
-\frac{1}{2 \tan \theta} \frac{d \ln v}{d \eta}+\frac{1}{\pi} \int_{0}^{\infty} \frac{d \ln v}{d \eta^{\prime}} \frac{\eta^{\prime}}{\eta^{\prime 2}-\eta^{2}} d \eta^{\prime}=\frac{a}{a^{2}+\eta^{2}} .
$$

The system of equations (2.14) - (2.15) allows us to determine the functions $v(\eta)$, $\lambda(\eta)$ together with the function $\theta(\eta)$ using Eq. (2.7). Once these functions are found, the velocity at point $D$, can be obtained from $v_{D}=\lim _{\eta \rightarrow \infty} v(\eta)$ and the angle of the tip of the splash jet, $\mu$ (see figure $2 a$ ) as

$$
\mu=\alpha_{-}+\alpha_{+}-\lim _{\eta \rightarrow \infty} \lambda(\eta)
$$

By choosing in the Bernoulli equation the location of the reference point at the stagnation point $A$, putting there $W\left(Z_{A}, t\right)=0$ or $w_{A}=0$, and taking advantage of the 
self-similarity of the flow, we can determine the pressure at any point of the flow region

$$
c_{p}^{*}=\frac{2\left(P-P_{A}\right)}{\rho V_{0}^{2}}=\Re\left(-2 w+2 z \frac{d w}{d z}\right)-\left|\frac{d w}{d z}\right|^{2},
$$

where the functions $z=z(\zeta), w=w(\zeta)$ and $d w / d z$ are determined from equations (2.2), (2.10) and (2.4), respectively.

\subsection{Direct impact of two 2-D rectangular jets}

It has been shown in Semenov \& Wu (2013) that when the liquid wedge hits a wall, the self similar solution tends to the steady solution of a rectangular jet impacting on the wall, when the angle of the wedge tends to zero and its flow rate remains finite. Here, the steady flow problem of two impinging rectangular 2- $D$ jets can be found to be a special case of the above self-similar solution. We notice that in this case the angles of the liquid wedges $\alpha_{+}, \alpha_{-}$and the angle of the splash jet $\mu$ are equal to zero. However, the length of the splash jet created from the impact of the rectangular jets is infinite. In addition, for steady flows velocity magnitude along the free surface is constant and the normal component of the velocity is zero, i.e. $v(\eta) \equiv v_{0}$ and $\lambda(\eta) \equiv 0$. By using these values the expressions for the complex velocity in Eq. (2.4) and for the derivative of the complex potential in Eq.(2.9) become

$$
\begin{gathered}
\frac{d w}{d z}=-i v_{0}\left(\frac{\zeta-a}{\zeta+a}\right) \\
\frac{d w}{d \zeta}=K \frac{\zeta^{2}-a^{2}}{\zeta\left(\zeta^{2}+1\right)} .
\end{gathered}
$$

The velocity field and geometry of the free boundaries can be determined by Eqs. (2.2) when the parameters $K$ and $a$ are known. They are determined from the conditions that flow rate across the upper jet is $q_{+}=h_{+} v_{0}$ and that across the lower jet is $q_{-}=h_{-} v_{0}$, where $h_{+}$and $h_{-}$are the widths of the upper and lower impinging jets, respectively. By integrating Eq. (2.19) along an arc of an infinitesimal circle in the parameter plane centered at point $P P^{\prime}(\zeta=0)$ and using the residue theorem we obtain

$$
q_{+}=\Im\left(\oint_{\zeta=0} \frac{d w}{d \zeta} d \zeta\right)=\frac{\pi}{2} \underset{\eta \rightarrow 0}{\operatorname{Res}} \frac{d w}{d \zeta} \zeta=-\frac{\pi}{2} K a^{2} .
$$

Similarly for the lower jet, integrating Eq. (2.19) along an arc of an infinitely large circle, we obtain

$$
q_{-}=\Im\left(\oint_{\zeta=\infty} \frac{d w}{d \zeta} d \zeta\right)=-\Im\left(\oint_{\zeta^{\prime}=0} \frac{d w}{d \zeta^{\prime}} \frac{1}{d \zeta^{\prime 2}} d \zeta^{\prime}\right)=-\frac{\pi}{2} K,
$$

where the new variable $\zeta^{\prime}=1 / \zeta$ is introduced to calculate the residue of the function $d w / d \zeta$ at infinity. From Eqs. (2.20) and (2.21) we obtain

$$
K=-\frac{2 q_{-}}{\pi}, \quad a^{2}=\frac{q_{+}}{q_{-}}=\frac{h_{+}}{h_{-}} .
$$

The angle of the splash jet $\beta$ and the argument of the velocity at the point $\zeta=i$ are the same. By taking the argument of expression (3.1) we determine the angle $\beta$ as follows

$$
\beta=-\arg \left(\left.\frac{d w}{d \zeta}\right|_{\zeta=i}\right)=-\frac{\pi}{2}+2 \tan ^{-1} \frac{1}{a} .
$$

In the case of equal jet widths, $h_{+}=h_{-}$, the parameter $a=1$, then the angle $\beta=0$, 
i.e. the outgoing splash jet is directed along the $x$ axis. These results are consistent with those presented in Milne-Thomson (1962).

\section{Results and discussion}

\subsection{Numerical approach}

The method of successive approximations for solving the system of nonlinear equations comprising the integro-differential equation (2.14) and the integral equation (2.15) is similar to that used by Semenov \& Iafrati (2006) and Semenov \& Cummings (2006) for solving the self-similar problem of water entry of a wedge and for the time-dependent Hele-Shaw flow with accounting effects of surface tension.

The numerical solution is sought on a fixed set of points $\eta_{j}, j=1, \ldots, 2 N$ distributed along the imaginary axis of the parameter plane. The total number of points is chosen in the range $N=100$ to 300 to check the convergence and accuracy of the solution procedure. The points $\eta_{j}$ are so distributed as to provide a higher density of the points $s_{j}=s\left(\eta_{j}\right)$ near the tip of the splash jet. Taking into account the singularities in equation (2.9) at points $\zeta=0, \zeta=i$, and $\zeta=\infty$, the location of the nodes nearest to these points is chosen as follows: $\eta_{1}=\varepsilon_{1}, \eta_{N-1}=1-\varepsilon_{1}, \eta_{N+1}=1+\varepsilon_{1}$, and $\eta_{2 N}=1 / \varepsilon_{1}$ where $\varepsilon_{1}=10^{-4}$ in order to provide a sufficiently good accuracy. The integrals in the system of equations are evaluated analytically after the linear interpolations of the functions $d \ln v / d \eta$ and $d \lambda / d \eta$ on the intervals $\left(\eta_{j-1}, \eta_{j}\right)$ are used.

\subsection{Validation of the numerical approach (symmetric liquid wedges)}

In figure 3, streamline patterns are shown for an upper liquid wedge of $\alpha_{+}=10^{0}$ and different angles $\alpha_{-}$of the lower liquid wedge. For the case shown in figure $3 a, \alpha_{+}=\alpha_{-}=$ $10^{0}$, figure $3 a$ clearly shows the symmetry of the flow and of the pressure distribution along the $y$-axis about the $x$-axis, which can be considered as a solid wall. The obtained value of the tip angle is $9.47^{\circ}$ which is close to the value $9.50^{\circ}$ obtained by Semenov \& $\mathrm{Wu}(2013)$ as the double contact angle from a different procedure for the problem of a liquid wedge impacting a solid wall.

\subsection{Vertical impact of two liquid wedges}

The streamline patterns for an upper liquid wedge of $\alpha_{+}=10^{0}$ colliding with liquid wedges of $\alpha_{-}=30^{\circ}, 70^{\circ}$, and $90^{\circ}$, respectively, are shown in figures $3 b-3 d$. For the case $\alpha_{-}=30^{\circ}$, it can be seen that the splash jet is directed into the half-plane of the liquid wedge of the smaller angle. For $x=0$, the $y$ coordinate of the tip of the upper undisturbed wedge(dotted line) is equal to -1 in figures $3 a-3 d$, which corresponds to the dimensionless velocity $v_{0}=1$ at point $P$ at infinity, while the tip coordinate of the lower undisturbed liquid wedge corresponding to the velocity at point $D$ is smaller than 1 , and it decreases as the angle $\alpha_{-}$increases. The undisturbed wedge-sides (dotted lines) in the self-similar coordinate system can be written as

$$
y_{+}=-1+\frac{1}{\tan \alpha_{+}} x, \quad y_{-}=v_{D}-\frac{1}{\tan \alpha_{-}} x .
$$

For $\alpha_{+}=10^{0}$ and $\alpha_{-}=70^{0}$ as illustrated in figure $3 c$, the splash jet touches the free surface of the upper liquid wedge creating a cavity. At the same time, the velocity direction of the liquid in the splash jet, which can be seen as the streamline slope, is almost parallel to the undisturbed free surface of the upper wedge. Due to the flow selfsimilarity, the cavity will continuously grow in the physical plane. In real flows in the presence of air, the pressure inside the closed growing cavity will become lower than that 


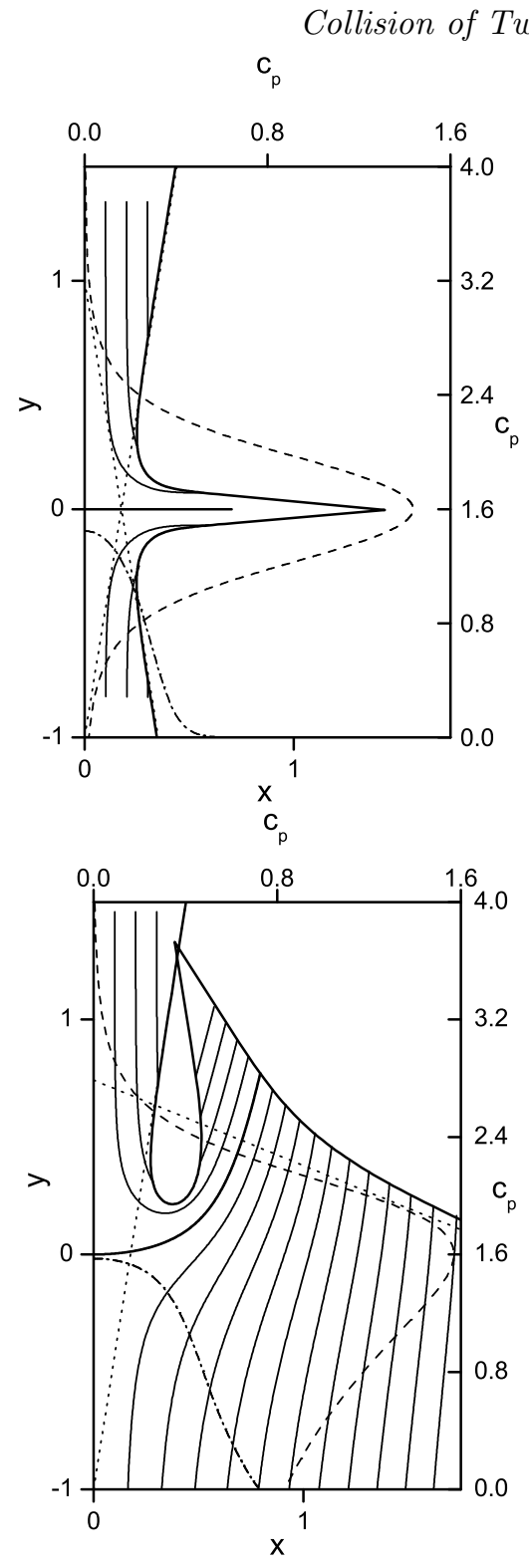

(a)

(b)

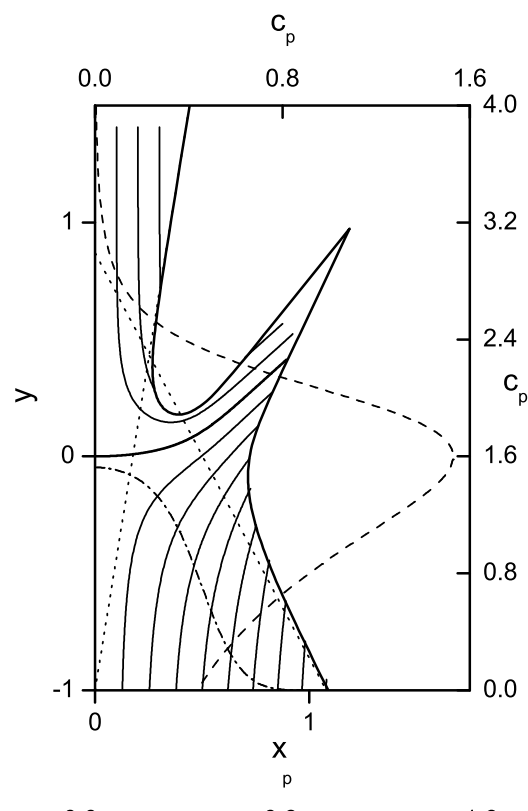

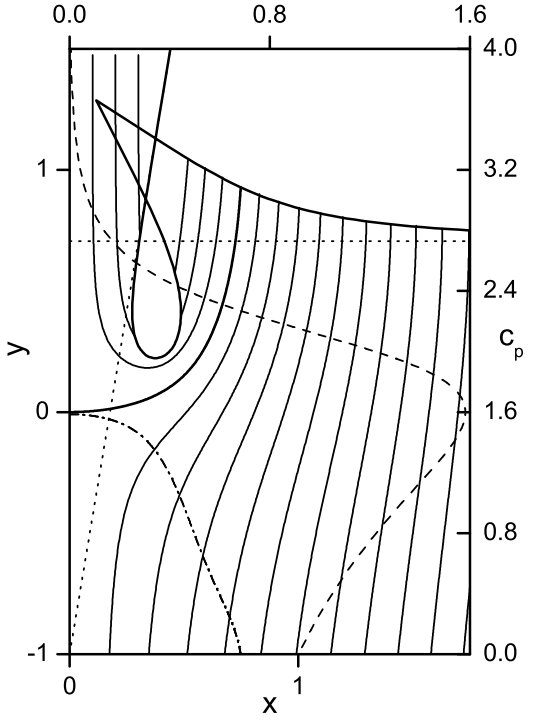

(c)

$(d)$

FiguRE 3. Streamline patterns (solid lines) in the self-similar plane and the pressure distribution along the y-axis (dashed line) and along the "zero" streamline starting at the origin (dot-dashed lines) for an upper liquid wedge of half-angle $10^{\circ}$ and a lower wedge of half-angles: (a) $10^{\circ}$, $v_{D}=1, v_{C}=1.45 ;(b) 30^{\circ}, v_{D}=0.87, v_{C}=1.53 ;$ (c) $70^{\circ}, v_{D}=0.74, v_{C}=1.39 ;(d) 90^{\circ}$ $v_{D}=0.70, v_{C}=1.29$. The increment of the stream function for two successive streamlines is 0.1 . The dotted lines show the undisturbed liquid wedges

on the free surface. The pressure difference between the two sides of the splash jet can push the splash jet towards the cavity, distort the splash jet, and make it possible for air to rush into the cavity. However, the present formulation for the problem does not consider such complicated flows. From a mathematical standpoint, the splash jet moves into the second sheet of the Riemann surface without interaction with the main flow.

For $\alpha_{+}=10^{\circ}$ and $\alpha_{-}=90^{\circ}$ shown in figure $3 d$, the splash jet is directed into the upper 


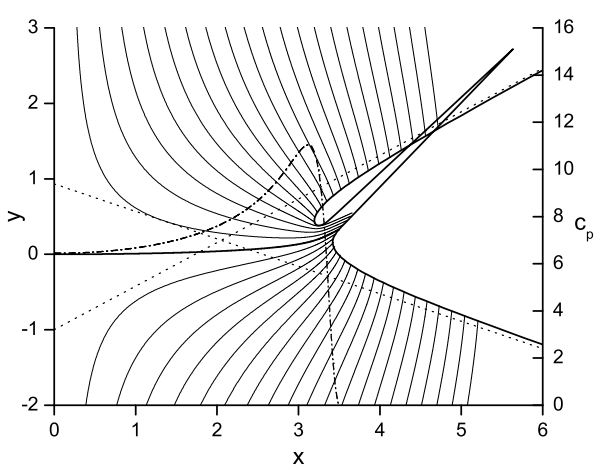

(a)

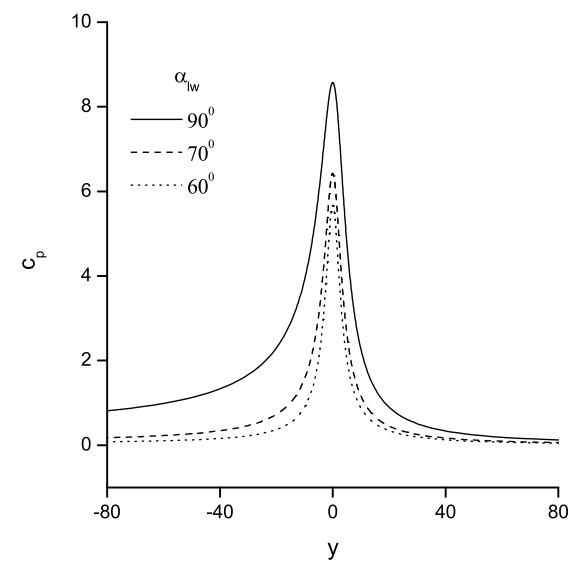

(c)

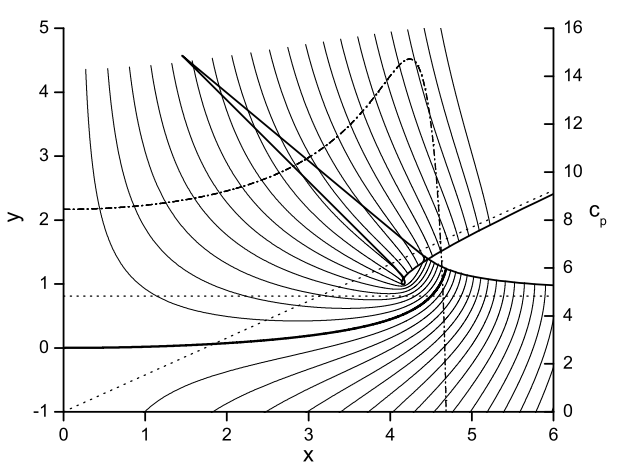

(b)

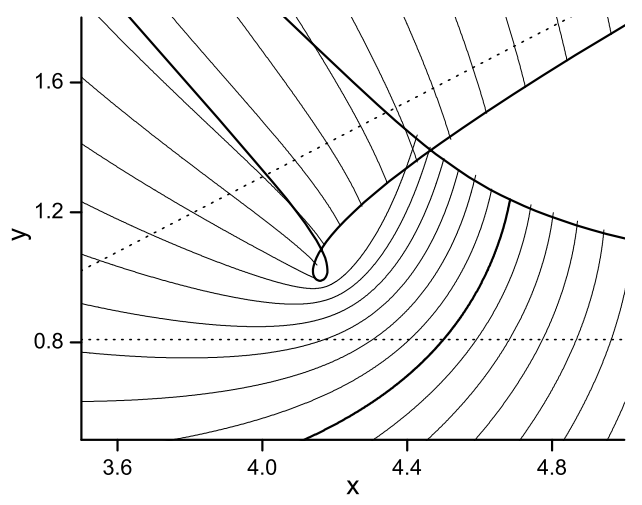

$(d)$

FIGURE 4. (a)-(b) streamline patterns (solid lines) in the self-similar plane and the pressure distribution along the "zero" streamline starting at the origin (dot-dashed lines) for an upper liquid wedge of $\alpha_{+}=60^{\circ}$ and a lower wedge of $(a) \alpha_{-}=70^{\circ}, v_{D}=0.94, v_{C}=6.25$, and $(b)$ $\alpha_{-}=90^{\circ}, v_{D}=0.81, v_{C}=4.91,(c)$ the pressure distribution along the $y$-axis, $(d)$ enlarged-scale flow pattern near the root of the splash jet for case $(b)$.

liquid wedge. The overlapping leads to a secondary impact between the splash jet and the wedge in physic reality. However, mathematically, the jet moves into the second sheet of the Riemann surface without interaction. In the case of overlapping of the splash jet and the wedge we can expect subsequent impacts and new splash jets in real situations.

Such multi-impact processes with the formation of multiple cavities facilitate the generation of a liquid/air mixture, liquid aeration, and the transformation of the splash jet into a spray. Similar situations occur for plunging breaking waves, as reviewed by Kiger and Duncan (2012), in which the splash jet formed from an impact between the wave crest and the free surface may be observed clearly in the case of oblique impacts or in the form of an air/liquid mixture in the case of nearly vertical impacts like a waterfall.

The streamline pattern for $\alpha_{+}=60^{\circ}$ and $\alpha_{-}=70^{\circ}$ is shown in figure $4 a$. It can be seen that the velocity at the tip of the splash jet becomes much higher than for a liquid wedge of angle $\alpha_{+}=10^{0}$ shown in figure $3 c$ while the angle of the splash jet becomes smaller. The splash jet overlaps with the undisturbed upper wedge, or moves into the second sheet the Riemann surface mathematically. However, the thickness of the splash jet becomes smaller in comparison with the cases shown in figure $3 c$. In physic reality, the distortion of the splash jet by the pressure difference between the growing cavity and 


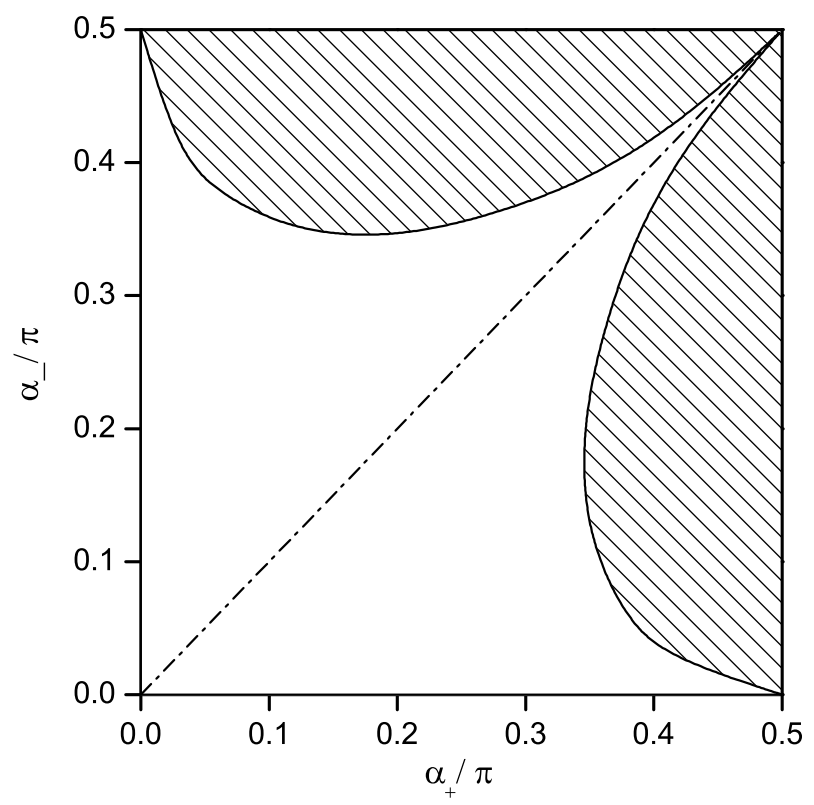

FiguRE 5. Flow regimes with and without secondary impact of the splash jet (shaded and opened area, respectively), in the plane of half-angles of the liquid wedges $\alpha_{+}$and $(a) \alpha_{-}$; the dot-dashed line corresponds to the symmetric flows with respect to $x$-axis.

the external free surface may destroy the splash jet, as discussed. The experiments of Thoroddsen (2002) showed an "ejecta sheet" as a spray at the initial stage of the impact of a drop onto a thin liquid film. From figure $4 b$ it can be seen that the splash jet is almost perpendicular to the free surface of the liquid wedge. The flow near the root of the splash jet is shown on an enlarged scale in figure $4 d$. The pressure along the "zero" streamline shown by a dot-dashed line increases as we move from the stagnation point to the core of the splash jet. In other words, the high-speed splash jet is caused by a high local pressure occurring near the intersection of the free surfaces of the liquid wedges. It occurs on the free surface of the liquid wedge of the larger angle.

The pressure distributions along the $y$-axis are shown in figure $4 c$ for an upper liquid wedge of angle $\alpha_{+}=60^{\circ}$ and for different angles of the lower liquid wedge including a flat free surface, $\alpha_{-}=90^{\circ}$. As illustrated, the pressure at the stagnation point increases substantially with the angle of the lower wedge. The pressure decays slowly for $y<0$, which corresponds to the wedge of the larger angle.

Flow regimes with and without secondary impact of the splash jet are shown in figure 5 by shaded and opened area, respectively, in the plane of half-angles of the liquid wedges $\alpha_{+}$and $\alpha_{-}$. It is seen that secondary impact always occurs if one of the liquid wedges has a flat free surface $\alpha_{ \pm} / \pi=0.5$. For the case $\alpha_{+}=\alpha_{-}$secondary impact of the splash jet does not occur for any angles $\alpha_{ \pm} / \pi<0.5$ since the flow is symmetric and the splash jet moves along the $x$-axis.

For the case of small deadrise angles shown in figures $4 a$ and $b$, the jet near its tip becomes very thin and the splash impacts the almost undisturbed side of the wedge. The evolution of the surface when $\alpha_{+} \rightarrow 0$ is shown in figure 6 for angles $\alpha_{+}=0.1^{0}, 0.5^{0}$, and $2^{0}$. It can be seen that as the angle $\alpha_{+}$decreases, the cavity becomes slender, while the splash jet tip angle and the velocity $v_{D}$ (shown by the coordinate of the horizontal dotted line) of the liquid in the lower half-plane increase. 


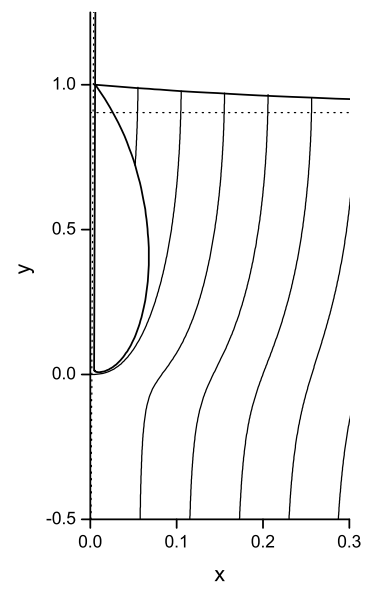

(a)

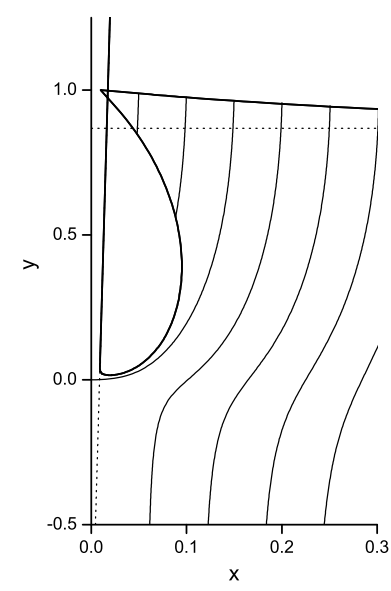

(b)

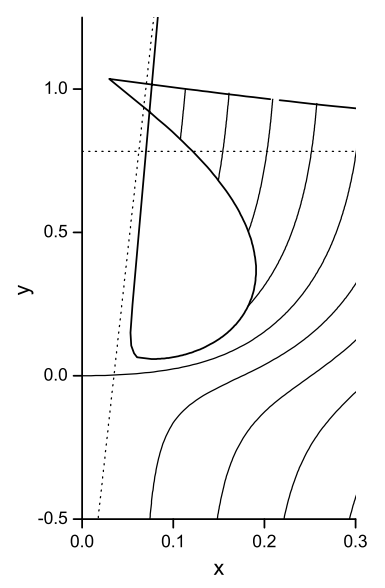

(c)

FIGURE 6. Streamline patterns in the self-similar plane for an upper liquid wedge at small angles: $(a) \alpha_{+}=0.1^{\circ}, v_{D}=0.9072, v_{C}=1.0000,(b) \alpha_{+}=0.5^{\circ}, v_{D}=0.8672, v_{C}=1.0001$ and (c) $\alpha_{+}=2^{\circ}, v_{D}=0.7830, v_{C}=1.0354$.

\begin{tabular}{cccccccccccc}
$\alpha_{+}$ & $\mu / \pi$ & $v_{C}$ & $\beta$ & $v_{D}$ & $c_{p A}$ & $\alpha_{+}$ & $\mu / \pi$ & $v_{C}$ & $\beta$ & $v_{D}$ & $c_{p A}$ \\
\hline 0.1 & 0.357 & 1.00002 & 89.69 & 0.907 & 1.015 & 30 & 0.090 & 2.094 & 79.21 & 0.722 & 2.974 \\
0.5 & 0.323 & 1.00008 & 89.43 & 0.867 & 1.032 & 40 & 0.0652 & 2.680 & 76.75 & 0.748 & 4.045 \\
2 & 0.259 & 1.035 & 88.35 & 0.783 & 1.125 & 50 & 0.0441 & 3.526 & 74.34 & 0.781 & 5.660 \\
5 & 0.211 & 1.128 & 86.74 & 0.730 & 1.301 & 60 & 0.0282 & 4.795 & 72.38 & 0.829 & 8.310 \\
10 & 0.169 & 1.292 & 84.83 & 0.705 & 1.583 & 70 & 0.0131 & 7.608 & 69.11 & 0.876 & 14.220 \\
20 & 0.122 & 1.653 & 81.82 & 0.704 & 2.199 & & & & & &
\end{tabular}

TABLE 1. Results for impact between the liquid wedge of angle $\alpha_{+}$and the flat free surface.

The angle and velocity of the tip of the splash jet, the velocity of the liquid in the lower half-plane, and the pressure at the stagnation point are shown in the following table for several angles $\alpha_{+}$.

\subsection{Different systems of coordinates}

In some cases it is more convenient to specify the ratio $V_{D} / V_{0}$ for example $V_{D} / V_{0}=1$, rather than choosing the stagnation point at the origin of the coordinate system. However, such formulation leads to additional nonlinear equation with respect to the velocity at point $D$ from which the coordinates of the stagnation point can be obtained. The present formulation can be used to obtain the flow configuration and velocity field for any ratio $V_{D} / V_{0}$. This is because the problem depends on only the relative velocity $V_{D}+V_{0}$, not $V_{D}$ and $V_{0}$ individually. Thus, the dynamic results such as pressure, and the kinematic results such flow field relative the stagnation point will not change if these velocities are changed to $V_{D}-d V$ and $V_{0}+d V$, respectively, where $d V$ is a transportation velocity of the moving system of coordinate in the physical plane.

To show that the mathematical formulation with such physical reality, we apply a transportation velocity $\Delta v$ in the self-similar plane to the total calculated flow, in which $v_{0}=v(0)=1$ and $v_{D}=\lim _{\eta \rightarrow \infty} v(\eta)$. The velocities at points $P$ and $D$ become, $v_{0}^{\prime}=v_{0}-\Delta v$ 
and $v_{D}^{\prime}=v_{D}+\Delta v$, respectively. The vertical coordinate of the stagnation point is now $y_{A}^{\prime}=\Delta v$ in the corresponding self-similarity plane $x^{\prime}-y^{\prime}$. By using the velocity at point $P$ as a reference velocity we may normalize the velocities and coordinates as follows

$$
v_{0}^{\prime \prime}=1, \quad x^{\prime \prime}=x \frac{1}{v_{0}-\Delta v}, \quad y^{\prime \prime}=y_{A}^{\prime \prime}+y \frac{1}{v_{0}-\Delta v},
$$

where

$$
\Delta v=\frac{v_{D}^{\prime \prime}-v_{D}}{v_{0}+v_{D}^{\prime \prime}}, \quad y_{A}^{\prime \prime}=\frac{\Delta v}{v_{0}-\Delta v}
$$

are obtained using the given ratio $V_{D} / V_{0}=v_{D}^{\prime \prime} / v_{0}^{\prime \prime}=v_{D}^{\prime \prime}$. The vertical coordinate $y_{A}^{\prime \prime}$ is the stagnation point in the self-similarity plane $x^{\prime \prime}-y^{\prime \prime}$, which corresponds to the given velocity ratio of the upper and lower liquid wedges.

\section{Conclusions}

The presented complete solution to the self-similar problem of an impact between two liquid wedges with the fully nonlinear boundary conditions revealed that the splash jet caused by the collision of two impacting mass of fluid is directed into the half-plane of the wedge with smaller angle and may form a closed cavity. The range of the wedge angles corresponding to the flows with and without overlapping of the splash jet with the main flow region has been determined. Along the axis of the flow symmetry, the pressure reaches its maximum at the stagnation point and decays more slowly in the wedge with the larger angle. In the case of small deadrise angles the maximum pressure in the flow region occurs near the root of the splash jet and causes the very thin and high speed splash jet.

This work is supported by Lloyd's Register Foundation (LRF) through the joint centre involving University College London, Shanghai Jiaotong University and Harbin Engineering University, to which the authors are most grateful. LRF supports the advancement of engineering-related education, and funds research and development that enhances safety of life at sea, on land and in the air.

\section{REFERENCES}

DAvidson, M.R. 2002 Spreading of an inviscid drop impacting on a liquid film. Chem. Eng. Sci. 57 3639-3647.

Howison, S.D., Ockendon, J.R., Oliver, J.M., Purvis, R. And Smith, F.T. 2005 Droplet impact on a thin fluid layer. J. Fluid Mech. $5421-23$.

Josserand, C. And Zaleski, S. 2003 Droplet splashing on a thin liquid film. Physics of Fluids 15(6) $1650-1657$.

Landrini, M., Colagrossi, A., Greco M., Tulin M.P. 2007. Gridless simulations of splashing processes and nearshore bore propagation. J. Fluid Mech. 591 183-213.

Keller, J.B. And Miksis, M.J 1983 Surface tension driven flows. SIAM J. Appl. Math. 43 268277

Keller, J.B., Milewski, P.A. And Vanden-Broeck, J.-M. 2002 Breaking and merging of liquid sheets and filaments. J. of Engn. Math. 42283290.

Kiger, K.T. And Duncan, J.H 2012 Air-Entrainment Mechanisms in Plunging Jets and Breaking Waves. Annu. Rev. Fluid Mech. 44 563-96.

Milne-Thomson, L.M. 1962 Theoretical Hydrodynamics. Macmillan \& Co Ltd.

OLIVER, J.M. 2007 Second-order Wagner theory for two-dimensional water-entry problems at small deadrise angles. J. Fluid Mech. 57259 - 85. 
Semenov, Y. A. \& Cummings, L. J. 2006 Free boundary Darcy flows with surface tension: analytical and numerical study. Euro. J. Appl. Math. 17, 607-631.

Semenov, Y.A. And IAfrati, A. 2006 On the nonlinear water entry problem of asymmetric wedges. J. Fluid Mech., 547, pp. $231-256$.

Semenov, Y.A. And Wu, G.X. 2013 Asymmetric impact between liquid and solid wedges. Proc $R$ Soc A 469: 20120203.

Thoroddsen, S.T. 2002 The ejecta sheet generated by the impact of a drop. J. Fluid Mech. $451373-381$.

Thoroddsen, S.T., Etoh, T.G. And Takehara, K. 2008 High-Speed Imaging of Drops and Bubbles. Annu. Rev. Fluid Mech. 40257 - 85.

Weiss, D.A. AND YARIN, A.L. 1999 Single drop impact onto liquid films: neck distortion, jetting, tiny bubble entrainment, and crown formation. J. Fluid Mech. 385229 - 254.

YARIN, A.L. 2006 Drop Impact Dynamics: Splashing, Spreading, Receding, Bouncing. . . AnNu. REV. Fluid MECH. 38159 - 92.

Zhang, L.V., Toole, J., FezzaA, K. and Deegan, R.D. 2012 Evolution of the ejecta sheet from the impact of a drop with a deep pool. J. Fluid Mech. $6905-15$. 